\title{
Agentes Comunitários de Saúde: uma perspectiva do capital social
}

\author{
Community Health Workers: a perspective of the social capital
}

Cleberson Williams dos Santos ${ }^{1}$

Milton Cordeiro Farias Filho ${ }^{2}$

${ }^{1}$ Fundação Getúlio Vargas. Av. 9 de Julho 2029, Bela

Vista. 01313-902 São Paulo SP Brasil.

cwsdireito@gmail.com

${ }^{2}$ Superintendência de Pesquisa, Universidade

da Amazônia. Belém PA

Brasil.

\begin{abstract}
This article reports on a survey of community health workers in Brazilian municipalities. The scope is to verify the influence of the network of social relations of agents in the daily work of the Family (FHS) teams. The theoretical base is addressing the social capital and the method is the analysis of social networks and their density measurements and EI-Index. In the data gathering, a questionnaire of the name generator type, with 266 agents in six municipalities in three different regions of Brazil (coded as Amazon 1 and 2, North Central 1 and 2, Central South 1 and 2) was used. Secondary data were also used. The results indicate that the profile of the community health agents is still a training limiter and they do not see themselves as a link between the community, the FHS teams and health facilities. The conclusion drawn is that the agents do not have the perception of their importance and that their internal networks have low density, with few external relations (other members of the FHS), which limits the expansion of social capital and hampers the dissemination of knowledge and experiences of actions of prevention.
\end{abstract}

Key words Community health agents, Social capital, Social networks
Resumo O artigo relata uma pesquisa com agentes comunitários de saúde em municípios brasileiros. Tem como objetivo verificar a influência das relações sociais em rede dos agentes no cotidiano de trabalho nas equipes de ESF. A base teórica é a abordagem do capital social e como método a análise de redes sociais e suas medidas de densidade e EI-Index. Na coleta de dados foi usado um questionário do tipo gerador de nomes, com 266 agentes, em seis municípios de três diferentes regiões do Brasil (codificados como Amazônico 1 e 2; Nordeste 1 e 2; Centro-Sul 1 e 2). Também foram usados dados secundários. Os resultados indicam que o perfil dos ACSs ainda é um limitador à capacitação e que estes não se veem como elo entre a comunidade, as equipes da ESF e as unidades de saúde. Conclui que os agentes não têm a percepção de sua importância e que suas redes internas apresentam baixa densidade, com poucas relações externas (outros membros da ESF), o que limita a ampliação do capital social e dificulta a difusão de conhecimentos e experiências de ações de prevenção.

Palavras-chave Agentes comunitários, Capital social, Redes sociais 


\section{Introdução}

O Programa Agente Comunitário de Saúde PACS, em nível nacional, tem origem no Programa Agente de Saúde do estado do Ceará, implantado em 1987, durante uma seca severa, ocasião em que foi contratada uma frente de mulheres ${ }^{1}$. Adotado nacionalmente, o PACS visa promover a reorientação da assistência à saúde, destinada à atenção básica (Portaria no $1.886^{2}$, Lei no $10.507^{3}$ ) e ser prestada pela gestão municipal do Sistema Único de Saúde (SUS).

A partir da implantação do PSF passou-se a privilegiar a atenção primária de caráter mais amplo. Sua ampliação fica evidente na consolidação da Estratégia Saúde da Família (ESF), que substitui o PSF, enquadrando em seus princípios os processos de trabalho baseados nos conceitos de prevenção de agravos, promoção da saúde, vigilância da saúde, integralidade e hierarquização, conectando-as à rede de serviços nos diversos níveis. Nesta nova concepção, o trabalho passa a ocorrer em microáreas de atuação, estabelecidas no cadastro de acompanhamento das famílias ${ }^{4,5}$.

A inserção do Agente Comunitário de Saúde (ACS) na ESF foi a forma encontrada pelo Ministério da Saúde para ampliar a capacidade de resolução do trabalho do $\mathrm{ACS}^{6}$. Trata-se de um modelo difundido pela Organização Mundial de Saúde (OMS) em diversos países, tendo como orientação a integração de serviços regionais aos sistemas nacionais, com a participação comunitária, relacionando a saúde ao desenvolvimento social e econômico ${ }^{7}$.

A ESF é a inserção de um modelo assistencial preventivo, orientado para a família em seu ambiente físico e social como núcleo de ação. Ela substitui o modelo tradicional curativo, focado na prevenção ${ }^{8}$. A ESF orienta o ACS a desenvolver suas atribuições no atendimento inicial dos indivíduos, na sua residência, levando informações ${ }^{9} \mathrm{e}$ reorganizando os processos de trabalho dos profissionais dos níveis hierárquicos mais elevados, em interação com a comunidade ${ }^{10}$. O modelo da ESF, com o papel do ACS tendo destaque, é o grande diferencial de prevenção em saúde de base comunitária, e faz do Programa brasileiro um dos maiores do mundo ${ }^{7}$.

A relevância das ações do ACS está na sua atuação como um elo entre a comunidade e as unidades locais do SUS ${ }^{11}$. Além disso, por ser morador da comunidade, como critério de admissão, o ACS deveria ter amplo conhecimento das necessidades comunitárias, pois compartilha seu ambiente cultural ${ }^{12}$, porém esta condição trás dificuldades para suas funções quando a comunidade não reconhece seu trabalho ${ }^{4}$.

A função e a relevância social do ACS foram formalizadas na Emenda Constitucional

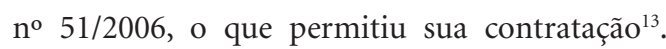
Esta orientação legal prevê que uma equipe da ESF deva fazer o acompanhamento de até 4.000 pessoas, contando com até 12 ACSs na equipe. Cada agente comunitário pode atender até 750 pessoas $^{8}$.

A informação é um dos requisitos básicos para a atividade do ACS, tanto na prevenção de doenças, quanto na promoção da saúde, em diversas atividades ${ }^{14}$. Sua integração à equipe multiprofissional é essencial por ele intermediar as necessidades dos moradores com as ações desses profissionais $^{15}$. Além disso, a interação com outros profissionais demonstrou que falta a ele o saber sistematizado e a utilização de instrumentos adequados ao trabalho, pois o que se tem como referência é o atendimento em situações estruturais precárias dos serviços de saúde ${ }^{16}$.

A previsão legal de o ACS ser morador da comunidade em que atua e, ao mesmo tempo, desenvolver as funções de assistência e promoção da saúde ${ }^{8,12}$, exige dele uma reputação capaz de transmitir confiança para comunidade que atende. Fato este que exige dele capacidade de manutenção e ampliação das relações sociais na comunidade e com os demais membros de sua equipe da $\mathrm{ESF}^{17}$.

Uma das formas de verificar as relações sociais no cotidiano de trabalho do ACS é a partir do capital social presente entre seus pares e membros das equipes da ESF. O capital social aqui compreendido como resultante das redes sociais formais e informais, estabelecidas pela confiança dos participantes, com usufruto de vantagens geradas pelo conhecimento, entre os quais a obtenção de informações ${ }^{18}$, além de outros recursos oriundos de ações instrumentais, o que faz do capital um ativo relacional mensurável ${ }^{19}$.

O capital social é derivado das relações estruturais existentes, de caráter multidimensional ${ }^{20}$, fundamentado em um conjunto de normas que afetam o bem estar da comunidade (neste caso, os integrantes das equipes de ESF). Este capital facilita a cooperação entre seus membros pela redução do custo de obtenção de informação.

Assim, o capital social do ACS se amplia de duas formas durante o exercício de suas funções: a) relações internas com seus pares (ACS-ACS), favorecendo trocas de experiências; b) relações externas com outros membros das equipes da ESF (ACS-ESF), o que amplia seus conhecimen- 
tos técnicos e auxilia nas ações de orientações e difusão de práticas de prevenção à saúde.

Esta tipologia na teoria de capital social pode ser estendida para as relações do ACS, em seu ambiente de trabalho nas equipes da ESF, da seguinte forma: a) capital social de ligação, caracterizado por relações entre pessoas, baseadas na confiança e cooperação ${ }^{20}$, para que se atinjam metas comuns aos participantes da rede. O exemplo são as relações entre os agentes no ambiente de trabalho; b) capital social ponte ${ }^{21}$, que aparece entre grupos semelhantes que buscam ações mútuas. O exemplo desse tipo é a relação entre os agentes e os técnicos de saúde; c) capital social de conexão $0^{22}$, baseado em relações de autoridade, mas com obtenção de recursos adicionais. Exemplo desse tipo são as relações dos agentes com médicos, enfermeiros, nutricionistas e outros profissionais de saúde de nível hierárquico superior.

Estudar redes sociais de agentes comunitários é uma forma de compreender os elementos de formação e desenvolvimento do capital social entre as equipes de trabalho da ESF. Isso favorece a percepção das contribuições do atributo relacional dos agentes e seu papel nas ações cotidianas de promoção da prevenção à saúde, portanto a base da atenção primária no campo da saúde coletiva. Diante deste contexto, o presente trabalho busca responder a seguinte questão: quais as influências das relações sociais de trabalho dos agentes comunitários de saúde no cotidiano das equipes da ESF?

A abordagem das redes sociais parte da compreensão de que estas são estruturas de troca de informações advindas de um conjunto de atores que se conectam por laços sociais, com a possibilidade de ampliação de capital social ${ }^{23}$. A unidade de análise é o ponto da rede, representado por pessoas, organizações ou estados. Estes são denominados de atores, e suas relações (ligações ou linhas) formam as redes ${ }^{24}$.

Dessa forma, o marco teórico da pesquisa é uma abordagem sustentada nas relações de confiança e cooperação ${ }^{20}$, em que as conexões ${ }^{22} \mathrm{e}$ pontes $^{21}$ se estabelecem para a formação do capital social. Por ser o ACS um profissional, cujo diferencial seria atuar como "elo" entre a comunidade e as unidades locais de gestão do SUS', ele está conectado a uma rede de serviços nos diversos níveis ${ }^{5}$, o que justifica ser estudado a partir de uma perspectiva de relações em redes pessoais de trabalho. Isso nos levou a buscar compreender a coesão entre os membros da rede por meio das medidas de densidade. Outro indicador foi o E-I
Index, usado para verificar relações internas e externas, servindo para estudar as relações entre os ACSs e entre estes e outros integrantes da equipe da ESF. Este índice é gerado no software específico para medir as entradas e saídas nas relações.

Diante dessa perspectiva, este trabalho busca preencher uma lacuna encontrada na literatura sobre o uso da análise de redes sociais em grupos de profissionais de atenção primária à saúde, a partir de uma perspectiva de capital social. Para isso, tem como objetivo verificar a influência das relações sociais em rede dos agentes no cotidiano de trabalho nas equipes de ESF.

\section{Método}

O trabalho teve início com a revisão da literatura, a partir de busca em bases de textos (Scielo, OMS -OPAS, Ebsco, Web of Science, Scopus e Medline). A seleção dos trabalhos que sustentam a discussão foi feita a partir de uma adaptação do modelo PRISMA $^{25}$.

No levantamento de campo foi usado um questionário do tipo gerador de nomes, comumente utilizado em pesquisas com o método de análise de redes sociais ${ }^{26}$. O questionário foi dividido em dois blocos: bloco I com questões sobre o perfil dos ACSs e bloco II sobre as condições de trabalho, relações entre os agentes e destes com outros profissionais das equipes da ESF. Para este bloco utilizou-se as redes de trabalho (relações específica para tratar de assuntos de trabalho), rede de confiança (relações estabelecidas tendo como base a confiança pessoal) e rede de competência (relações baseadas no reconhecimento de competência) ${ }^{26}$.

A coleta de dados foi feita mediante contatos prévios pessoalmente, via telefone e por e-mail, ocorrendo de três formas: a) questionários preenchidos nas visitas em reuniões e assembleias realizadas pelos agentes; b) questionários entregues aos responsáveis pelos agentes, com a devolução feita por ocasião de apresentação dos mapas de produção por estes; c) preenchimento e devolução dos questionários nas comunidades em que os agentes atuavam e/ou nas unidades de saúde a que estavam vinculados.

Informações complementares foram retiradas de outras fontes: Confederação Nacional dos Agentes Comunitários; Conselho Nacional de Secretarias Municipais de Saúde; Organização Mundial de Saúde; coordenadores da atenção básica; secretários municipais e representantes dos ACSs. 
A seleção dos municípios se baseou na divisão geoeconômica dos complexos regionais: a) amazônico; b) nordeste; c) centro-sul ${ }^{27}$. Foram selecionados dois municípios por complexo, com população na faixa de 30 mil a 120 mil habitantes $^{28}$ e priorizados os mais próximos das capitais, em virtude da maior acessibilidade dos pesquisadores. Dos 39 municípios que atendiam aos critérios da faixa populacional e proximidade com as capitais, 16 aceitaram participar da pesquisa, porém apenas em seis destes os questionários foram inteiramente respondidos. Estes tiveram os nomes codificados (Tabela 1) para sua não identificação.

Os resultados das redes sociais ${ }^{24}$ dos ACSs foram analisados no software Ucinet/Netdraw versão $6.232^{29}$ e são apresentados em forma de medidas de densidade e E-I Index ${ }^{30}$. As duas medidas são para verificar a intensidade e os tipos de relacionamentos entre grupos diferentes (integrantes da ESF).

As medidas de densidade mostram que quanto mais contatos recíprocos existirem em uma rede, mais informações serão compartilhadas entre os atores sobre o que estão realizando. Tratase de uma medida da proporção entre "laços efe- tivos" (relações entre membros da rede) e "laços possíveis" (relações possíveis de se estabelecer). A densidade mostra que quanto mais densa é a rede (mais próxima de 1,0), mais uniformizados são os contatos entre os pontos (atores). Uma densidade baixa (inferior a 0,2 ) indica uma rede dispersa e com baixa coesão interna $a^{24,29}$.

O E-Index é um índice gerado no software, a partir das relações (laços) e é resultado da diferença entre laços internos e externos, dividido pelo total de laços: os valores mais próximos de +1 indicam tendência de relacionamentos externos ao grupo; já os valores mais próximos de -1 indicam tendência de relacionamentos inter$\operatorname{nos}^{30}$. É uma medida que ajuda a verificar tendências de difusão de informações entre grupos.

\section{Resultados e Discussão}

Os resultados mostram que, quanto ao perfil (Tabela 2), predominam ACSs do sexo feminino $(84,2 \%)$. Este fato tem relação com suas origens ${ }^{1}$, tendência que vem se mantendo ${ }^{31}$, também por ser este sexo predominante entre os profissionais de saúde.

Tabela 1. Municípios de Abrangência da Pesquisa.

\begin{tabular}{lccccc}
\hline \multicolumn{1}{c}{ Município } & Habitantes $^{28}$ & No $^{\text {de ACSs }}{ }^{32}$ & Questionários & Habitantes/ACS & Equipes de ESF \\
\hline Amazônico 1 (Pará) & 35.557 & 115 & 44 & $309 / 1$ & 11 \\
Amazônico 2 (Pará) & 54.417 & 96 & 55 & $566 / 1$ & 7 \\
Nordeste 1 (Alagoas) & 49.853 & 107 & 34 & $465 / 1$ & 15 \\
Nordeste 2 (Alagoas) & 71.834 & 113 & 45 & $605 / 1$ & 18 \\
Centro-Sul 1 (Santa Catarina) & 120.926 & 73 & 40 & $1.656 / 1$ & 14 \\
Centro-Sul 2 (Santa Catarina) & 39.740 & 76 & 48 & $522 / 1$ & 12 \\
Total & & 580 & 266 & & \\
\hline
\end{tabular}

\begin{tabular}{|c|c|c|c|c|c|c|c|}
\hline Idade & $\%$ & Escolaridade & $\%$ & Tempo como ACS & $\%$ & Sexo & $\%$ \\
\hline De 35 a 44 & 44,0 & Ens. Med. Completo & 66,9 & 5 a10 anos & 81,2 & Feminino & 84,2 \\
\hline De 25 a 34 & 31,6 & Ens. Sup. Incompleto & 11,7 & Menos de lano & 8,6 & Masculino & 15,8 \\
\hline De 45 a 59 & 19,5 & Ens. Med. Incompleto & 6,8 & 3 a 4 anos & 5,6 & Total & 100,0 \\
\hline De 21 a 24 & 1,9 & Ens. Sup. Completo & 6,8 & 1 a 2 anos & 4,1 & & \\
\hline De 60 a 69 & 1,9 & Ens. Fund. Completo & 6,0 & Mais de 11 anos & 0,4 & & \\
\hline De 18 a 20 & 0,8 & Ens. Fund. Incompleto & 1,9 & Total & 100,0 & & \\
\hline Mais de 70 & 0,4 & Sem Escolaridade & 0,0 & & & & \\
\hline Total & 100,0 & Total & 100,0 & & & & \\
\hline
\end{tabular}

Fonte: Elaboração própria com dados da pesquisa. 
A maior parte apresenta escolaridade de nível médio $(66,9 \%)$. Já quanto à faixa etária, a maioria $(75,6 \%)$ tem entre 25 e 44 anos. Esta é a faixa etária mais afetada pelo desemprego entre os profissionais com baixa qualificação, confirmando outros trabalhos que defendem que a atividade de ACS também é uma saída para o desemprego, ainda que tenha ocorrido uma melhoria na escolarização ${ }^{33}$. Quanto ao tempo na atividade, a maioria $(81,2 \%)$ tem entre 5 a 10 anos.

O principal motivo para os ACSs ingressarem na função foi "oportunidade de trabalho" $(49,2 \%)$, o que tem relação com a atividade exercida anteriormente: vendedor $(31,6 \%)$, desempregado $(15,8 \%)$, estudante $(15,4 \%)$ e auxiliares em serviços administrativos (10,9\%).

$O$ perfil e as dificuldades estruturais de trabalho relatadas pelos ACSs mostram os desafios para a gestão da ESF, em termos de treinamentos, capacitação, atualização e incentivos para valorização profissional. Além disso, o tempo na função revela que a compreensão de seu papel como agente é construído nas práticas cotidianas ${ }^{34}$. A atuação como ACS mostra um lado positivo que é a possibilidade de maior aprendizagem com a experiência acumulada na atividade de saúde e ampliação de informações sobre a comunidade, sendo esta a maior contribuição para a ESF.

As dificuldades estruturais enfrentadas pelos ACSs podem ter relação com alguns problemas na gestão da ESF, que geram questionamentos sobre a efetividade deles. Um dos problemas é o distanciamento entre os agentes e os demais membros da ESF, verificado nas análises das redes, por meio do E-I Index. Portanto, a unidade de análise é a relação entre os membros da rede de profissionais integrantes da ESF, tendo como referência central os ACSs.
Na opinião dos ACSs (Tabela 3) o fator “ajudar a comunidade em que moram" é considerado por eles um fator positivo no exercício da função $(65,4 \%)$. Já como fator negativo para a função aparece a "falta de estrutura e de recursos" (44,0\%), e a falta de "valorização profissional” (24,1\%). Esses fatores negativos têm relação com as respostas sobre "dificuldades enfrentadas diariamente no trabalho". Neste caso, as maiores dificuldades relatadas foram: "falta de apoio ao trabalho" (35,3\%) e problemas de "acesso e segurança” para desenvolver o trabalho (10,5\%).

A condição de morador da comunidade também traz dificuldades na execução de suas funções, o que é confirmado por trabalhos recentes que indicam o não reconhecimento desta função e de seu papel ${ }^{4}$. Este fato foi confirmado por $97 \%$ dos ACSs entrevistados em três municípios. Logo, ficou demonstrado que os ACSs não se reconhecem como elo entre a comunidade e os profissionais das equipes da ESF ou com as unidades locais de saúde. Fato este que contraria o argumento de que o ACS é um elo comunitário ${ }^{9}$.

Essa dificuldade de percepção do seu papel, das funções e dos problemas estruturais que enfrentam se reflete no que os ACSs relatam como desvalorização profissional por parte da gestão municipal da saúde. Isto reforça a ideia de que enfrentam condições adversas, quando comparado com outros profissionais da equipe da ESF, pois carecem de um saber sistematizado e da utilização de instrumentos adequados ao seu trabalho ${ }^{16}$. Além disso, muitas vezes, os ACSs relatam que suas funções estão restritas às orientações gerais de prevenção e marcação de consultas para os comunitários nas unidades de saúde. Tal fato contribui para a ineficiência do PACS e da ESF, já que a função do ACS é a que têm maior visibilidade ${ }^{2,3}$.

Tabela 3. Percepção dos ACSs sobre os fatores positivos e negativos da função.

\begin{tabular}{lrlr}
\hline \multicolumn{1}{c}{ Fatores Positivos } & \multicolumn{1}{c}{$\%$} & \multicolumn{1}{c}{ Fatores Negativos } & $\%$ \\
\hline Ajudar. Informar à comunidade & 65,4 & Falta de estrutura/Recursos & 44,0 \\
Identificação com a função & 12,4 & Falta de valorização/Reconhecimento & 24,1 \\
Conhecimento e aprendizado & 6,4 & Baixa remuneração & 11,7 \\
Reconhecimento e valorização & 6,0 & Nenhum & 7,1 \\
Elo: comunidade-unidade & 3,0 & Não encontrar os moradores & 4,5 \\
Nenhum & 2,6 & Outros & 3,8 \\
Tipo de trabalho/Jornada de trabalho & 2,3 & Pouca resolutividade & 2,6 \\
Morar na comunidade & 1,9 & Jornada de trabalho excessiva & 2,3 \\
Total & 100,0 & Total & 100,0 \\
\hline
\end{tabular}

Fonte: Elaboração própria com dados da pesquisa. 
Os agentes relataram que as dificuldades enfrentadas diariamente no trabalho estão ligadas a estrutura e recursos, segurança e acesso para desenvolver suas atividades, confirmando que são vários os problemas já relatados em outros $\operatorname{artigos}^{4,15,16}$.

As redes de maior densidade são as que transferem mais informações para os ACSs. Estas podem chegar aos demais membros das equipes da ESF, porém o atributo principal é a confiança nos membros. Assim, os resultados (Tabela 4) indicam que as menores densidades (0.009) foram encontradas nas redes de confiança dos Agentes do município Amazônico 1 (Pará). Já a maior densidade (0.081) foi encontrada no município Centro-Sul 1 (Santa Catarina). Neste município, os ACSs apresentaram maior escolaridade e menor número de reclamações das condições de trabalho.

Os resultados das medidas de densidade estão associados à presença do capital social de ligação, por se tratar de uma relação entre pessoas, baseada em confiança e cooperação para que se atinjam metas comuns aos participantes da rede ${ }^{20}$. Também há evidências do tipo capital social ponte, que aparece entre grupos semelhantes que buscam ações mútuas ${ }^{21}$.

Nas redes estudadas, a maior densidade significa maior circulação de conhecimentos e informações, o que se reflete em melhores condições de desenvolver as atividades voltadas para os objetivos da prevenção baseada em orientações, que é o papel primordial do ACS. Suas relações de trabalho, formais e informais, estabelecidas a partir da confiança dos participantes ${ }^{20}$ permitem o usufruto de vantagens geradas pelo conhecimento, especialmente a obtenção de informações ${ }^{21}$, além de outros recursos e ações instrumentais, resultando em capital social como um ativo relacional. Portanto, teoricamente, a abordagem do capital social explica a capilaridade dos agentes na comunidade e sua relevância profissional nas equipes da ESF.

A ausência das interações nas redes sociais de trabalho leva a espaços vazios ou buracos estruturais $^{24,29}$, reduzindo o fluxo dos recursos e o acesso ao capital social da rede ${ }^{24}$. Este é o caso do município Nordeste 2 (Alagoas), com menor densidade nas redes de trabalho, confiança e competência. Logo, as redes dos ACSs desse município apresentam baixa confiança, pouco reconhecimento de competência entre os participantes e baixa circulação de informações, o que significa que o capital social presente não está auxiliando o bem-estar da comunidade, nem facilitando a cooperação entre os seus membros. Aparentemente neste caso, a esperada redução do custo de obtenção de informação $0^{20}$, que faz parte da essência da ESF, não ocorreu. Da mesma forma que a estrutura da rede deste município não comprova parte das predições teóricas aqui trabalhadas ${ }^{20,21,23}$.

No que se refere às relações entre membros dos grupos, os que apresentam maior número de relações internas entre seus pares diminuem a possibilidade de acesso a novas informações ${ }^{21}$. As relações externas aumentam a possibilidade de ampliar os conhecimentos advindos de outros grupos. Já as relações internas limitam o acesso a novas informações de fora do grupo ${ }^{19,29,30}$. Para verificação desse fenômeno e de seus significados para a atividade de trabalho dos grupos de ACS e da ESF o E-I Index se mostrou mais adequado (Tabela 3). Este índice usa a relação como unidade de análise e calcula sua origem (interna e externa) para definição de grupos mais abertos e mais fechados a relacionamentos.

Os resultados (Tabela 5) mostram um E-I Index negativo para os grupos com maior número de ligações internas. O que significa maior número de relações entre seus pares (ACS-ACS). Estas são relações do tipo laços fortes e significa maior

Tabela 4. Densidade nas Redes de Trabalho, Confiança e Competência.

\begin{tabular}{lccc}
\hline \multicolumn{1}{c}{ Municípios } & Rede de Trabalho & Rede de Confiança & Rede de Competência \\
\hline Amazônico 1 (Pará) & 0.010 & 0.009 & 0.042 \\
Amazônico 2 (Pará) & 0.007 & 0.050 & 0.039 \\
Nordeste 1 (Alagoas) & 0.013 & 0.029 & 0.030 \\
Nordeste 2 (Alagoas) & 0.007 & 0.029 & 0.026 \\
Centro-Sul 1 (Santa Catarina) & 0.008 & 0.081 & 0.066 \\
Centro-Sul 2 (Santa Catarina) & 0.012 & 0.062 & 0.049 \\
\hline
\end{tabular}

Fonte: Elaboração própria com dados da pesquisa. 
Tabela 5. E-I Index nas Redes de Confiança e Competência

\begin{tabular}{|c|c|c|c|c|c|c|}
\hline \multirow{3}{*}{ Municípios } & \multicolumn{3}{|c|}{ Rede Confiança } & \multicolumn{3}{|c|}{ Rede Competência } \\
\hline & \multicolumn{2}{|c|}{ Densidade } & \multirow[b]{2}{*}{ E-I Index } & \multicolumn{2}{|c|}{ Densidade } & \multirow[b]{2}{*}{ E-I Index } \\
\hline & Inter. & Exter. & & Inter. & Exter. & \\
\hline Amazônico 1 (Pará) & 0.034 & 0.033 & -0.103 & 0.029 & 0.033 & 0.024 \\
\hline Amazônico 2 (Pará) & 0.038 & 0.015 & -0.836 & 0.031 & 0.022 & -0.437 \\
\hline Nordeste 1 (Alagoas) & 0.063 & 0.032 & -0.158 & 0.052 & 0.032 & -0.453 \\
\hline Nordeste 2 (Alagoas) & 0.044 & 0.029 & -0.172 & 0.042 & 0.031 & -0.008 \\
\hline Centro-Sul 1 (Santa Catarina) & 0.051 & 0.020 & -0.492 & 0.026 & 0.046 & 0.459 \\
\hline Centro-Sul 2 (Santa Catarina) & 0.044 & 0.039 & 0.006 & 0.036 & 0.032 & 0.182 \\
\hline
\end{tabular}

Fonte: Elaboração própria com dados da pesquisa.

cooperação para a redução do custo de obter informações internas ${ }^{21,29}$ (ACS-ACS). Inversamente, há uma elevação dos custos de obtenção de informações oriundas das relações externas ${ }^{29,30}$ (ACS-Outros membros da ESF).

Os dois municípios cujas redes apresentam mais ligações externas são: Centro-Sul 2 (Santa Catarina) para as redes de confiança e competência; Centro-Sul 1 (Santa Catarina) e Amazônico 1 (Pará) apenas para as redes de competência. Nos demais municípios as redes apresentam uma forte ligação interna (ACS-ACS), o que significa que os ACSs dos outros quatro municípios têm baixa interação com os demais membros das equipes da ESF, ocorrendo limitada transferência de informações e conhecimentos sobre suas experiências na função, especialmente o aprendizado resultante da interação com outros profissionais da ESF.

Quando os agentes comunitários estabelecem ligações com grupos de técnicos de enfermagem e/ou técnicos administrativos, o capital social é do tipo ponte. Mesmo com aumento das ligações externas, haverá a manutenção da horizontalidade, pois os grupos ainda continuam no mesmo nível hierárquico ${ }^{21}$. Trata-se de uma relação de aprendizado dos conhecimentos acumulados por outros profissionais e de fácil transferência para a comunidade. No entanto, esta foi a relação com menor frequência entre os grupos estudados.

Quando as relações externas dos agentes se estabelecem com outros profissionais da equipe da ESF que estão em níveis hierárquicos superiores, ocorre um capital social do tipo conexão, pois estes ocupam posições de autoridade, o que, de acordo com a teoria, permite melhor intermediação de recursos adicionais ${ }^{22}$.

Dessa forma, quanto maior a interatividade entre os grupos, horizontal ou vertical, de acordo com suas conexões externas, o E-I Index terá valores positivos, o que indicará maior cooperação e acesso a novas informações entre os grupos. Portanto, o que os grupos de ACSs estudados revelaram foi uma baixa relação com outros profissionais da ESF, pois prevaleceu o E-I Index negativo na maioria das redes estudadas. Este resultado mostra que há um limitador na difusão de conhecimento técnico e de troca de informações relevantes entre os membros da ESF, que são as poucas relações entre seus membros, o que de certa forma compromete a efetividade da ESF.

\section{Conclusão}

O perfil dos ACSs revelou que a baixa escolaridade e as atividades exercidas antes, influenciaram a entrada na função. Este fato é semelhante nos diferentes municípios, independente do número de habitantes e das características socioeconômicas.

A pouca percepção de seu papel na prevenção à saúde, aliado à baixa capacidade de respostas das equipes da ESF, especialmente pelas dificuldades estruturais das unidades de saúde, mostram um desgaste da imagem social da função do ACS. Estes fatos influenciam na reputação do agente na comunidade. Isso faz com que ele não se veja como elo entre comunidade e demais membros das equipes da ESF e as unidades de saúde.

O fato de ser morador da comunidade não é, em si, uma condição para o ACS ampliar sua atuação na prevenção aos problemas de saúde da comunidade. Ele pode até compreender bem esses problemas, mas não pode ajudar a resolvê-los, mesmo que sua função gere expectativas na comunidade. As condições estruturais em que atua é o principal limitador.

A baixa densidade nas redes de relações sociais no ambiente de trabalho é um indicador de 
pouca coesão entre os ACSs. Nas redes sociais dos agentes prevalecem relações internas (ACS-ACS) com pouca interação externa (ACS-Outros da ESF). Isso impede que o ACS aprenda mais com os outros profissionais da equipe da ESF, o que reduz sua capacidade de transferir informações de prevenção à saúde, limitando o efeito multiplicador que representa na atenção primária de saúde.

\section{Colaboradores}

CW dos Santos e MC Farias Filho contribuíram igualmente para a construção do trabalho, desde seu planejamento até a versão final. 


\section{Referências}

1. Avila MMM. O Programa de agentes comunitários de saúde no Ceará: o caso de Uruburetama. Cien Saude Colet 2011; 16(1):349-360.

2. Brasil. Portaria ${ }^{\circ}$. 1.886 , de 18 de dezembro de 1997. Aprova as normas e diretrizes do Programa de Agente Comunitário e do Programa de Saúde da Família e dá outras providências. Diário Oficial da União 1997; 22 dez.

3. Brasil. Lei $\mathrm{n}^{\circ} 10.507$, de 10 de julho de 2002. Cria a profissão de Agente Comunitário de Saúde e dá outras providências. Diário Oficial da União 2002; 11 jul.

4. Acioli S, Keblian LVA. A visita domiciliar de enfermeiros e agentes comunitários de saúde da estratégia saúde da família. Rev Eletr Enf 2014; 16(1):161-169.

5. Vidal SV, Motta LCDS, Gomes AP, Siqueira-Batista R. Problemas bioéticos na estratégia saúde da família: reflexões necessárias. Rev Bioética 2014; 22(2):347-357.

6. Turrini RNT, Lebrão ML, Cesar CLG. Resolutividade dos serviços de saúde por inquérito domiciliar: percepção do usuário. Cad Saude Publica 2008; 24(3):663-674.

7. Lehmann U, Sanders D. Comunity healt wokers: what do we know about them? Geneva: WHO; 2007.

8. Brasil. Portaria no 2.488, de 21 de outubro de 2011 Aprova a Política Nacional de Atenção Básica, estabelecendo a revisão de diretrizes e normas para a organização da Atenção Básica para o Programa Saúde da Família (PSF) e o Programa Agentes Comunitários de Saúde (PACS). Diário Oficial da União 2011; 24 out.

9. De Melo Costa S, Araújo FF, Martins LV, Nobre LLR, Araújo FM, Rodrigues CAQ. Agente comunitário de saúde: elemento nuclear das ações em saúde. Cien Saude Colet 2013; 18(7):2147-2156.

10. Fracolli LA, Gomes MFP, Gryschek ALDFP. Percepções de gestores municipais sobre ações de promoção da saúde: em foco os agentes comunitários de saúde. Saúde Soc 2014; 23(3):919-927.

11. Mascarenhas CHM, Prado FO, Fernandes MH. Fatores associados à qualidade de vida de agentes comunitários de saúde. Cien Saude Colet 2013; 18(5):1375-1386.

12. Jardim TA, Lancman S. Aspectos subjetivos de morar e trabalhar na mesma comunidade: a realidade vivenciada pelo agente comunitário de saúde. Interface (Botucatu) 2009; 13(28):123-135.

13. Alexandrino M, Vicente P. Direito constitucional descomplicado. 11a ed. São Paulo: Método; 2013.

14. Staliano P, Araujo TCCF. Promoção da saúde e prevenção de doenças: um estudo com agentes comunitários de saúde. Rev Psicol Saúde 2011; 3(1):43-51.

15. Marzari CK, Junges JR, Selli L. Agentes comunitários de saúde: perfil e formação. Cien Saude Colet 2011; 16(1):873-880.

16. Santos LPGS, Fracolli LA. Community Health Aides: possibilities and limits to health promotion. Rev Esc Enferm 2010; 44(1):76-83.

17. Fortuna CM, Mishima SM, Matumoto S, Pereira MJB. O trabalho de equipe no programa de saúde da família: reflexões a partir de conceitos do processo grupal e de grupos operativos. Rev Latino-Am Enfermagem 2005; 13(2):262-268.

18. Putnan RD. Democracies in flux: the evolution of social capital in contemporary society. Oxford: Oxford University Press; 2002.
19. Lin N. A network theory of social capital. In: Castiglione D, Deth JV, Wolleb G, editors. The handbook of social capital. Oxford: Ed. Oxford; 2008. p. 69.

20. Fukuyama F. A grande ruptura: a natureza humana e a reconstituição da ordem social. Rio de Janeiro: Rocco; 2000.

21. Marteleto R, Silva ABO. Redes e capital social: o enfoque da informação para o desenvolvimento local. Ci Inf 2004; 33(3):45-49.

22. Grootaert C, Narayan D, Jones VN, Woolcock M. Questionário integrado para medir capital social (QI-MCS). Washington: Banco Mundial; 2003.

23. Tomaél M I, Marteleto RM. Redes sociais: posições dos atores no fluxo da informação. Encontros Bibli 2007; 11(n. esp. 1):75-91.

24. Hanneman RA, Riddle M. Introduction to social network methods. Berkeley: University of California; 2005.

25. Moher D, Liberati A, Tetzlaff J, Altman DG; PRISMA Group. Preferred reporting items for systematic reviews and meta-analyses: the PRISMA statement. Int $J$ Surg 2010; 8(5):336-341.

26. Requena Santos F. Redes sociales y cuestionários. Madrid: Centro de Investigaciones Sociológicas; 1996.

27. Geiger PP. Organização regional do Brasil. Rev Geográfica 1964; 61:51.

28. Instituto de Geografia e Estatística. Estimativa da população residente nos municípios brasileiros, 2013. [acessado 2015 abr 13]. Disponível em: http://www. ibge.gov.br/home/estatistica/populacao/estimativa2013/estimativa_dou.shtm

29. Borgatti SP, Everett MG, Freeman LC. Ucinet 6 for Widows: software for social network analysis. Massachusetts: Harvard Analytic Technologies; 2005.

30. Krackhardt D, Stern RN. Informal networks and organizational crises: an experimental simulation. Social Psychology Quarterly 1988; 51(2):123-140.

31. Silva MHS, Santos MR. Perfil de atividades dos agentes comunitários de saúde vinculados ao programa de saúde da família da zona norte de Juiz de Fora. Rev. APS 2007; 8(2):109-117.

32. Brasil. Ministério da Saúde (MS). Secretaria de Atenção à Saúde. Diretoria de Atenção Básica. [acessado 2015 fev 23]. Disponível em: http://dab.saude.gov.br/portaldab/esus.php?conteudo=esus

33. Mota RA, David HMSL. A crescente escolarização do ACS: uma introdução do processo de trabalho? Trab Educ Saúde 2010; 8(2):229-248.

34. Ferraz L, Aerts DRGC. O cotidiano de trabalho do agente comunitário de saúde em Porto Alegre. Cien Saude Colet 2005; 10(2):347-355.

Artigo apresentado em 29/06/2015

Aprovado em 17/12/2015

Versão final apresentada em 19/12/2015 
